\title{
COUPLED FIXED POINT THEOREMS WITH $C$-CLASS FUNCTIONS
}

\author{
Arslan H. Ansari, Murat Ozdemir, Mohammad S. Khan and Isa Yildirim
}

\begin{abstract}
In this paper, we introduce a new type of coupled fixed point theorem with $C$-class functions in partially ordered complete metric space.
\end{abstract}

Keywords: Coupled fixed point; Partially ordered set; Mixed monotone mappings, $C$ class function.

\section{Introduction and Preliminaries}

Fixed point theory plays an important role in various applications of many branches of mathematics. During the last several years, there have been many generalizations of the concept of metric spaces to study metrical fixed point theory. The first result in metric spaces endowed with a partial ordering was obtained by Ran and Reurings [9]. They presented some applications in solving matrix equations. In 2005, Nieto and Lopez [7] extended the result of Ran and Reurings [8], for non-decreasing mappings and applied their result to get a unique solution for a first order differential equation. Bhaskar and Lakshmikantham [5] obtained some coupled fixed point theorems for mixed monotone operators using certain contractive-type condition defined on a partially ordered metric space. Later, Berinde [4] extended the work of Bhaskar and Lakshmikantham [5] by significantly weakening the involved contractive condition.

In order to state the main result in this paper, we need the following notions.

Definition 1.1. Let $(X, \leq)$ be a partially ordered set such that the product space $X \times X$ is endowed with the following partial order:

$$
\text { for }(x, y),(u, v) \in X \times X, \quad(u, v) \leq(x, y) \Leftrightarrow x \geq u, y \leq v \text {. }
$$

Received March 21, 2017; accepted March 13, 2018

2010 Mathematics Subject Classification. Primary 54H10; Secondary 54H25 
We say that a mapping $F: X \times X \rightarrow X$ has the mixed monotone property if $F(x, y)$ is monotone non-decreasing in $x$ and is monotone non-increasing in $y$, that is, for any $x, y \in X$,

$$
x_{1}, x_{2} \in X, x_{1} \leq x_{2} \Rightarrow F\left(x_{1}, y\right) \leq F\left(x_{2}, y\right)
$$

and

$$
y_{1}, y_{2} \in X, y_{1} \leq y_{2} \Rightarrow F\left(x, y_{1}\right) \geq F\left(x, y_{2}\right) .
$$

Definition 1.2. An element $(x, y) \in X \times X$ is called a coupled fixed point of the mapping $F: X \times X \rightarrow X$ if

$$
F(x, y)=x \text { and } F(y, x)=y .
$$

The following result is due to Bhaskar and Lakshmikantham[3].

Theorem 1.1. [5] Let $(X, \leq)$ be a partially ordered set and suppose there is a metric $d$ on $X$ such that $(X, d)$ is a complete metric space. Let $F: X \times X \rightarrow X$ be a continuous mapping having the mixed monotone property on $X$. Assume that there exists a constant $k \in[0,1)$ with

$$
d(F(x, y), F(u, v)) \leq \frac{k}{2}[d(x, u)+d(y, v)], \forall x \geq u, \quad y \leq v .
$$

If there exist $x_{0}, y_{0} \in X$ such that

$$
x_{0} \leq F\left(x_{0}, y_{0}\right) \text { and } y_{0} \geq F\left(y_{0}, x_{0}\right),
$$

then there exist $x, y \in X$ such that

$$
x=F(x, y) \text { and } y=F(y, x) .
$$

In [5] Bhaskar and Lakshmikantham also established some uniqueness results for coupled fixed points, as well as the existence of fixed points of $F$ ( $x$ is a fixed point of $F$ if $F(x, x)=x)$. More recently, Yildirim [11] obtained the following generalization of Theorem 1.3.

Theorem 1.2. [11] Let $(X, \leq)$ be a partially ordered set and suppose there is a metric $d$ on $X$ such that $(X, d)$ is a complete metric space. Let $F: X \times X \rightarrow X$ be a continuous mapping having the mixed monotone property on $X$. Assume that $F$ satisfies the following condition:

$$
d(F(x, y), F(u, v)) \leq \delta(x, y, u, v)[d(x, u)+d(y, v)]
$$

where

$$
\delta(x, y, u, v)=\frac{d(x, F(u, v))+d(y, F(v, u))+d(u, F(x, y))+d(v, F(y, x))}{1+2[d(x, F(x, y))+d(y, F(y, x))+d(u, F(u, v))+d(v, F(v, u))]}
$$


for all $x, y, u, v \in X$ with $x \geq u$ and $y \leq v$.

If there exist $x_{0}, y_{0} \in X$ such that

$$
x_{0} \leq F\left(x_{0}, y_{0}\right) \text { and } y_{0} \geq F\left(y_{0}, x_{0}\right),
$$

then

a) F has at least a coupled fixed point $(x, y) \in X \times X$ such that

$$
x=F(x, y) \text { and } y=F(y, x) .
$$

b) if $(x, y),(u, v)$ are two distinct coupled fixed points of $F$, then $d(x, u)+$ $d(y, v) \geq \frac{1}{4}$.

Definition 1.3. [2] A mapping $f:[0, \infty)^{2} \rightarrow \mathbb{R}$ is called a $C$-class function if it is continuous and satisfies the following axioms:

(1) $f(s, t) \leq s$

(2) $f(s, t)=s$ implies that either $s=0$ or $t=0$; for all $s, t \in[0, \infty)$.

The letter $\mathcal{C}$ will denote the class of all $C$-class functions.

Example 1.1. [2] The following functions $f:[0, \infty)^{2} \rightarrow \mathbb{R}$ are elements of $\mathcal{C}$, for all $s, t \in[0, \infty)$ :

(1) $f(s, t)=s-t, f(s, t)=s \Rightarrow t=0$;

(2) $f(s, t)=m s, 0<m<1, f(s, t)=s \Rightarrow s=0$;

(3) $f(s, t)=\frac{s}{(1+t)^{r}} ; r \in(0, \infty), f(s, t)=s \Rightarrow s=0$ or $t=0$;

(4) $f(s, t)=\log \left(t+a^{s}\right) /(1+t), a>1, f(s, t)=s \Rightarrow s=0$ or $t=0$;

(5) $f(s, t)=\ln \left(1+a^{s}\right) / 2, a>e, f(s, t)=s \Rightarrow s=0$;

(6) $f(s, t)=(s+l)^{\left(1 /(1+t)^{r}\right)}-l, l>1, r \in(0, \infty), f(s, t)=s \Rightarrow t=0$;

(7) $f(s, t)=s \log _{t+a} a, a>1, f(s, t)=s \Rightarrow s=0$ or $t=0$;

(8) $f(s, t)=s-\left(\frac{1+s}{2+s}\right)\left(\frac{t}{1+t}\right), f(s, t)=s \Rightarrow t=0$;

(9) $f(s, t)=s \beta(s), \beta:[0, \infty) \rightarrow[0,1)$, and is continuous, $f(s, t)=s \Rightarrow s=0$;

(10) $f(s, t)=s-\frac{t}{k+t}, f(s, t)=s \Rightarrow t=0$;

(11) $f(s, t)=s-\varphi(s), f(s, t)=s \Rightarrow s=0$, here $\varphi:[0, \infty) \rightarrow[0, \infty)$ is a continuous function such that $\varphi(t)=0 \Leftrightarrow t=0$;

(12) $f(s, t)=\operatorname{sh}(s, t), f(s, t)=s \Rightarrow s=0$, here $h:[0, \infty) \times[0, \infty) \rightarrow[0, \infty)$ is a continuous function such that $h(t, s)<1$ for all $t, s>0$;

(13) $f(s, t)=s-\left(\frac{2+t}{1+t}\right) t, f(s, t)=s \Rightarrow t=0$;

(14) $f(s, t)=\sqrt[n]{\ln \left(1+s^{n}\right)}, f(s, t)=s \Rightarrow s=0$;

(15) $f(s, t)=\phi(s), f(s, t)=s \Rightarrow s=0$,here $\phi:[0, \infty) \rightarrow[0, \infty)$ is a continuous function such that $\phi(0)=0$, and $\phi(t)<t$ for $t>0$;

(16) $f(s, t)=\frac{s}{(1+s)^{r}} ; r \in(0, \infty), f(s, t)=s \Rightarrow s=0$. 
Definition 1.4. [[6]] A function $\psi:[0, \infty) \rightarrow[0, \infty)$ is called an altering distance function if the following properties are satisfied:

(i) $\psi$ is non-decreasing and continuous,

(ii) $\psi(t)=0$ if and only if $t=0$,

(iii) $\psi(s+t)<\psi(s)+\psi(t)$ for $s, t>0$,

Remark 1.1. We denote the set of altering distance functions by $\Psi$.

Definition 1.5. An ultra altering distance function is a continuous, non-decreasing mapping $\varphi:[0, \infty) \rightarrow[0, \infty)$ such that $\varphi(t)>0, t>0$ and $\varphi(0) \geq 0$.

Remark 1.2. We denote the set of ultra altering distance functions by $\Phi_{u}$.

Definition 1.6. A tripled $(\psi, \varphi, f)$, where $\psi \in \Psi, \varphi \in \Phi_{u}$ and $f \in \mathcal{C}$ is said to be monotone if for any $x, y \in[0, \infty)$, we have

$$
x \leqslant y \Longrightarrow f(\psi(x), \varphi(x)) \leqslant f(\psi(y), \varphi(y)) .
$$

Example 1.2. Let $f(s, t)=s-t$ and $\phi(x)=\sqrt{x}$

$$
\psi(x)=\left\{\begin{array}{ll}
\sqrt{x} & \text { if } 0 \leq x \leq 1, \\
x^{2}, & \text { if } \mathrm{x}>1
\end{array} .\right.
$$

Then $(\psi, \phi, f)$ is monotone.

Example 1.3. Let $f(s, t)=s-t, \phi(x)=x^{2}$

$$
\psi(x)=\left\{\begin{array}{ll}
\sqrt{x} & \text { if } 0 \leq x \leq 1, \\
x^{2}, & \text { if } \mathrm{x}>1
\end{array} .\right.
$$

Then $(\psi, \phi, f)$ is not monotone.

We will use the following lemma in order to prove our main result.

Lemma 1.1. [3] Suppose that $(X, d)$ is a metric space and $\left\{x_{n}\right\}$ is a sequence in $X$ such that $d\left(x_{n}, x_{n+1}\right) \rightarrow 0$ as $n \rightarrow \infty$. If $\left\{x_{n}\right\}$ is not a Cauchy sequence then there exist an $\epsilon>0$ and sequences of positive integers $\{m(k)\}$ and $\{n(k)\}$ with $m(k)>n(k)>k$ such that $d\left(x_{m(k)}, x_{n(k)}\right) \geq \epsilon, d\left(x_{m(k)-1}, x_{n(k)}\right)<\epsilon$ and

(i) $\lim _{k \rightarrow \infty} d\left(x_{m(k)}, x_{n(k)}\right)=\epsilon$;

(ii) $\lim _{k \rightarrow \infty} d\left(x_{m(k)-1}, x_{n(k)}\right)=\epsilon$;

(iii) $\lim _{k \rightarrow \infty} d\left(x_{m(k)}, x_{n(k)-1}\right)=\epsilon$.

Inspired by the work of earlier mathematician, in this paper we study coupled fixed point theorems for continuous mapping $F: X \times X \rightarrow X$ having the mixed monotone property on $X$ with $C$-class functions in partially ordered complete metric space. 


\section{Main Results}

Now we are in a position to state and prove the main results of this paper.

Theorem 2.1. Let $(X, \leq)$ be a partially ordered set and suppose there is a metric $d$ on $X$ such that $(X, d)$ is a complete metric space. Let $F: X \times X \rightarrow X$ be a continuous mapping having the mixed monotone property on $X$. Assume that $F$ satisfies the following condition:

$$
\begin{aligned}
\psi(d(F(x, y), F(u, v))) \leq & \frac{1}{2} f(\psi(\delta(x, y, u, v)[d(x, u)+d(y, v)]) \\
& \varphi(\delta(x, y, u, v)[d(x, u)+d(y, v)])))
\end{aligned}
$$

where $\psi \in \Psi, \varphi \in \Phi_{u}, f \in C$, such that $(\psi, \varphi, f)$ is monotone and a constant $k \in[1,2]$ with

$$
\delta(x, y, u, v)=\frac{1+k[d(x, F(u, v))+d(y, F(v, u))+d(u, F(x, y))+d(v, F(y, x))]}{1+2[d(x, F(x, y))+d(y, F(y, x))+d(u, F(u, v))+d(v, F(v, u))]}
$$

for all $x, y, u, v \in X$ with $x \geq u$ and $y \leq v$.

If there exist $x_{0}, y_{0} \in X$ such that

$$
x_{0} \leq F\left(x_{0}, y_{0}\right) \text { and } y_{0} \geq F\left(y_{0}, x_{0}\right),
$$

then, $F$ has at least a coupled fixed point $(x, y) \in X \times X$ such that

$$
x=F(x, y) \text { and } y=F(y, x) .
$$

Proof. a) Suppose that the two sequences $\left\{x_{n}\right\}$ and $\left\{y_{n}\right\}$ in $X$ such that,

$$
x_{n+1}=F\left(x_{n}, y_{n}\right) \text { and } y_{n+1}=F\left(y_{n}, x_{n}\right)
$$

for all $n=0,1,2, \ldots$

Now, we claim that $\left\{x_{n}\right\}$ is non-decreasing and $\left\{y_{n}\right\}$ is non-increasing i.e.,

$$
x_{n} \leq x_{n+1} \text { and } y_{n} \geq y_{n+1}
$$

for all $n=0,1,2, \ldots$ From the hypotheses of Theorem 2.1 , we know that $x_{0}, y_{0} \in X$ with

$$
x_{0} \leq F\left(x_{0}, y_{0}\right) \text { and } y_{0} \geq F\left(y_{0}, x_{0}\right)
$$

From the mixed monotone property of $F$, we have

$$
x_{1}=F\left(x_{0}, y_{0}\right) \text { and } y_{1}=F\left(y_{0}, x_{0}\right) .
$$

This implies that $x_{0} \leq x_{1}$ and $y_{0} \geq y_{1}$. That is, the inequalities (2.3) are true for $n=0$. We suppose that $x_{n} \leq x_{n+1}$ and $y_{n} \geq y_{n+1}$ for some $n$. Now we will prove 
that (2.3) is true for $n+1$. Indeed, from (2.3) and the mixed monotone property of $F$, we have

$$
x_{n+2}=F\left(x_{n+1}, y_{n+1}\right) \geq F\left(x_{n}, y_{n+1}\right) \geq F\left(x_{n}, y_{n}\right)=x_{n+1}
$$

and

$$
y_{n+2}=F\left(y_{n+1}, x_{n+1}\right) \leq F\left(y_{n}, x_{n+1}\right) \leq F\left(y_{n}, x_{n}\right)=y_{n+1} .
$$

From induction, $x_{n} \leq x_{n+1}$ and $y_{n} \geq y_{n+1}$ for all $n$. Since (2.1), $x_{n-1} \leq x_{n}$ and $y_{n-1} \geq y_{n}$, we obtain

$$
\begin{aligned}
& \psi\left(d\left(F\left(x_{n}, y_{n}\right), F\left(x_{n-1}, y_{n-1}\right)\right)\right) \\
& \leq \frac{1}{2} f\left(\psi \left(\left(\begin{array}{c}
1+k\left[\begin{array}{c}
d\left(x_{n}, F\left(x_{n-1}, y_{n-1}\right)\right)+d\left(y_{n}, F\left(y_{n-1}, x_{n-1}\right)\right) \\
+d\left(x_{n-1}, F\left(x_{n}, y_{n}\right)\right)+d\left(y_{n-1}, F\left(y_{n}, x_{n}\right)\right)
\end{array}\right] \\
1+2\left[\begin{array}{c}
d\left(x_{n}, F\left(x_{n}, y_{n}\right)\right)+d\left(y_{n}, F\left(y_{n}, x_{n}\right)\right) \\
+d\left(x_{n-1}, F\left(x_{n-1}, y_{n-1}\right)\right)+d\left(y_{n-1}, F\left(y_{n-1}, x_{n-1}\right)\right)
\end{array}\right]
\end{array}\right)\right.\right. \\
& \left.\left[d\left(x_{n}, x_{n-1}\right)+d\left(y_{n}, y_{n-1}\right)\right]\right), \\
& \varphi\left(\left(\begin{array}{c}
1+k\left[\begin{array}{c}
d\left(x_{n}, F\left(x_{n-1}, y_{n-1}\right)\right)+d\left(y_{n}, F\left(y_{n-1}, x_{n-1}\right)\right) \\
+d\left(x_{n-1}, F\left(x_{n}, y_{n}\right)\right)+d\left(y_{n-1}, F\left(y_{n}, x_{n}\right)\right)
\end{array}\right] \\
1+2\left[\begin{array}{c}
d\left(x_{n}, F\left(x_{n}, y_{n}\right)\right)+d\left(y_{n}, F\left(y_{n}, x_{n}\right)\right) \\
+d\left(x_{n-1}, F\left(x_{n-1}, y_{n-1}\right)\right)+d\left(y_{n-1}, F\left(y_{n-1}, x_{n-1}\right)\right)
\end{array}\right]
\end{array}\right)\right. \\
& \left.\left.\left[d\left(x_{n}, x_{n-1}\right)+d\left(y_{n}, y_{n-1}\right)\right]\right)\right) \\
& =\frac{1}{2} f\left(\psi\left(\left(\begin{array}{c}
d\left(x_{n}, x_{n}\right)+d\left(y_{n}, y_{n}\right) \\
k \frac{+d\left(x_{n-1}, x_{n+1}\right)+d\left(y_{n-1}, y_{n+1}\right)}{1+2\left[\begin{array}{c}
d\left(x_{n}, x_{n+1}\right)+d\left(y_{n}, y_{n+1}\right) \\
+d\left(x_{n-1}, x_{n}\right)+d\left(y_{n-1}, y_{n}\right)
\end{array}\right]}
\end{array}\right)\left[d\left(x_{n}, x_{n-1}\right)+d\left(y_{n}, y_{n-1}\right)\right]\right),\right. \\
& \left.\varphi\left(\left(\begin{array}{c}
d\left(x_{n}, x_{n}\right)+d\left(y_{n}, y_{n}\right) \\
k \frac{+d\left(x_{n-1}, x_{n+1}\right)+d\left(y_{n-1}, y_{n+1}\right)}{1+2\left[\begin{array}{c}
d\left(x_{n}, x_{n+1}\right)+d\left(y_{n}, y_{n+1}\right) \\
+d\left(x_{n-1}, x_{n}\right)+d\left(y_{n-1}, y_{n}\right)
\end{array}\right]}
\end{array}\right)\left[d\left(x_{n}, x_{n-1}\right)+d\left(y_{n}, y_{n-1}\right)\right]\right)\right) \\
& =\frac{1}{2} f\left(\psi\left(\left(\frac{1+k\left[d\left(x_{n-1}, x_{n+1}\right)+d\left(y_{n-1}, y_{n+1}\right)\right]}{1+2\left[\begin{array}{c}
d\left(x_{n}, x_{n+1}\right)+d\left(y_{n}, y_{n+1}\right) \\
+d\left(x_{n-1}, x_{n}\right)+d\left(y_{n-1}, y_{n}\right)
\end{array}\right]}\right)\left[d\left(x_{n}, x_{n-1}\right)+d\left(y_{n}, y_{n-1}\right)\right]\right)\right. \text {, }
\end{aligned}
$$




$$
\begin{aligned}
& \left.\left(\left(\frac{1+k\left[d\left(x_{n-1}, x_{n+1}\right)+d\left(y_{n-1}, y_{n+1}\right)\right]}{1+2\left[\begin{array}{c}
d\left(x_{n}, x_{n+1}\right)+d\left(y_{n}, y_{n+1}\right) \\
+d\left(x_{n-1}, x_{n}\right)+d\left(y_{n-1}, y_{n}\right)
\end{array}\right]}\right)\left[d\left(x_{n}, x_{n-1}\right)+d\left(y_{n}, y_{n-1}\right)\right]\right)\right) \\
\leq & \frac{1}{2} f\left(\psi\left(\left[\begin{array}{c}
1+k\left[\begin{array}{c}
d\left(x_{n-1}, x_{n}\right)+d\left(x_{n}, x_{n+1}\right) \\
+d\left(y_{n-1}, y_{n}\right)+d\left(y_{n}, y_{n+1}\right)
\end{array}\right] \\
1+2\left[\begin{array}{c}
d\left(x_{n}, x_{n+1}\right)+d\left(y_{n}, y_{n+1}\right) \\
+d\left(x_{n-1}, x_{n}\right)+d\left(y_{n-1}, y_{n}\right)
\end{array}\right]
\end{array}\right)\left[d\left(x_{n}, x_{n-1}\right)+d\left(y_{n}, y_{n-1}\right)\right]\right),\right. \\
& \left.\left(\left[\begin{array}{c}
1+k\left[\begin{array}{c}
d\left(x_{n-1}, x_{n}\right)+d\left(x_{n}, x_{n+1}\right) \\
+d\left(y_{n-1}, y_{n}\right)+d\left(y_{n}, y_{n+1}\right)
\end{array}\right) \\
1+2\left[\begin{array}{c}
d\left(x_{n}, x_{n+1}\right)+d\left(y_{n}, y_{n+1}\right) \\
+d\left(x_{n-1}, x_{n}\right)+d\left(y_{n-1}, y_{n}\right)
\end{array}\right]
\end{array}\right)\left[d\left(x_{n}, x_{n-1}\right)+d\left(y_{n}, y_{n-1}\right)\right]\right)\right)
\end{aligned}
$$

This implies

$$
\begin{aligned}
& \psi\left(d\left(x_{n+1}, x_{n}\right)\right) \\
& \leq \frac{1}{2} f\left(\psi \left(\left(\frac{1+k\left[d\left(x_{n-1}, x_{n}\right)+d\left(x_{n}, x_{n+1}\right)+d\left(y_{n-1}, y_{n}\right)+d\left(y_{n}, y_{n+1}\right)\right]}{1+2\left[d\left(x_{n}, x_{n+1}\right)+d\left(y_{n}, y_{n+1}\right)+d\left(x_{n-1}, x_{n}\right)+d\left(y_{n-1}, y_{n}\right)\right]}\right)\right.\right. \\
& {\left.\left[d\left(x_{n}, x_{n-1}\right)+d\left(y_{n}, y_{n-1}\right)\right]\right), } \\
& \varphi\left(\left(\frac{1+k\left[d\left(x_{n-1}, x_{n}\right)+d\left(x_{n}, x_{n+1}\right)+d\left(y_{n-1}, y_{n}\right)+d\left(y_{n}, y_{n+1}\right)\right]}{1+2\left[d\left(x_{n}, x_{n+1}\right)+d\left(y_{n}, y_{n+1}\right)+d\left(x_{n-1}, x_{n}\right)+d\left(y_{n-1}, y_{n}\right)\right]}\right)\right. \\
&\left.\left.(2.6) \quad\left[d\left(x_{n}, x_{n-1}\right)+d\left(y_{n}, y_{n-1}\right)\right]\right)\right) .
\end{aligned}
$$

Similarly, from (2.1), $y_{n-1} \geq y_{n}$ and $x_{n-1} \leq x_{n}$, we have

$$
\begin{aligned}
& \psi\left(d\left(y_{n+1}, y_{n}\right)\right) \\
\leq & \frac{1}{2} f\left(\psi \left(\left(\frac{1+k\left[d\left(y_{n-1}, y_{n}\right)+d\left(y_{n}, y_{n+1}\right)+d\left(x_{n-1}, x_{n}\right)+d\left(x_{n}, x_{n+1}\right)\right]}{1+2\left[d\left(y_{n}, y_{n+1}\right)+d\left(x_{n}, x_{n+1}\right)+d\left(y_{n-1}, y_{n}\right)+d\left(x_{n-1}, x_{n}\right)\right]}\right)\right.\right. \\
& {\left[d\left(y_{n}, y_{n-1}\right)+d\left(x_{n}, x_{n-1}\right)\right], } \\
& \varphi\left(\left(\frac{1+k\left[d\left(y_{n-1}, y_{n}\right)+d\left(y_{n}, y_{n+1}\right)+d\left(x_{n-1}, x_{n}\right)+d\left(x_{n}, x_{n+1}\right)\right]}{1+2\left[d\left(y_{n}, y_{n+1}\right)+d\left(x_{n}, x_{n+1}\right)+d\left(y_{n-1}, y_{n}\right)+d\left(x_{n-1}, x_{n}\right)\right]}\right)\right. \\
& {\left.\left.\left[d\left(y_{n}, y_{n-1}\right)+d\left(x_{n}, x_{n-1}\right)\right]\right)\right) . }
\end{aligned}
$$

From these inequalities (2.6) and (2.7), we obtain 
$(2.8)$

$$
\begin{aligned}
& \psi\left(d\left(x_{n+1}, x_{n}\right)+d\left(y_{n+1}, y_{n}\right)\right) \\
\leq & \psi\left(d\left(x_{n+1}, x_{n}\right)\right)+\psi\left(d\left(y_{n+1}, y_{n}\right)\right) \\
\leq & f\left(\psi \left(\left(\frac{1+k\left[d\left(y_{n-1}, y_{n}\right)+d\left(y_{n}, y_{n+1}\right)+d\left(x_{n-1}, x_{n}\right)+d\left(x_{n}, x_{n+1}\right)\right]}{1+2\left[d\left(y_{n}, y_{n+1}\right)+d\left(x_{n}, x_{n+1}\right)+d\left(y_{n-1}, y_{n}\right)+d\left(x_{n-1}, x_{n}\right)\right]}\right)\right.\right. \\
& {\left[d\left(y_{n}, y_{n-1}\right)+d\left(x_{n}, x_{n-1}\right)\right] } \\
& \varphi\left(\left(\frac{1+k\left[d\left(y_{n-1}, y_{n}\right)+d\left(y_{n}, y_{n+1}\right)+d\left(x_{n-1}, x_{n}\right)+d\left(x_{n}, x_{n+1}\right)\right]}{1+2\left[d\left(y_{n}, y_{n+1}\right)+d\left(x_{n}, x_{n+1}\right)+d\left(y_{n-1}, y_{n}\right)+d\left(x_{n-1}, x_{n}\right)\right]}\right)\right. \\
& {\left.\left.\left[d\left(y_{n}, y_{n-1}\right)+d\left(x_{n}, x_{n-1}\right)\right]\right)\right) } \\
\leq & \psi\left(\left(\frac{1+k\left[d\left(y_{n-1}, y_{n}\right)+d\left(y_{n}, y_{n+1}\right)+d\left(x_{n-1}, x_{n}\right)+d\left(x_{n}, x_{n+1}\right)\right]}{1+2\left[d\left(y_{n}, y_{n+1}\right)+d\left(x_{n}, x_{n+1}\right)+d\left(y_{n-1}, y_{n}\right)+d\left(x_{n-1}, x_{n}\right)\right]}\right)\right. \\
& {\left.\left[d\left(y_{n}, y_{n-1}\right)+d\left(x_{n}, x_{n-1}\right)\right]\right) . }
\end{aligned}
$$

So

$$
d\left(x_{n+1}, x_{n}\right)+d\left(y_{n+1}, y_{n}\right) \leq d\left(y_{n}, y_{n-1}\right)+d\left(x_{n}, x_{n-1}\right) .
$$

Put $\lambda_{n+1}=d\left(x_{n+1}, x_{n}\right)+d\left(y_{n+1}, y_{n}\right)$. Then $\left\{\lambda_{n}\right\}$ is a non-increasing sequence of real numbers and must converge to $\lambda \geq 0$. Letting $n \rightarrow \infty$ in (2.8), we have that

$$
\psi(\lambda) \leq f\left(\psi\left(\frac{1+2 k \lambda}{1+4 \lambda} \times \lambda\right), \varphi\left(\frac{1+2 k \lambda}{1+4 \lambda} \times \lambda\right)\right) \leq \psi\left(\frac{1+2 k \lambda}{1+4 \lambda} \times \lambda\right) \leq \psi(\lambda) .
$$

So, $\psi\left(\frac{1+2 k \lambda}{1+4 \lambda} \times \lambda\right)=0$, or, $\varphi\left(\frac{1+2 k \lambda}{1+4 \lambda} \times \lambda\right)=0$. Hence $\lambda=0$. Then

$$
\lim _{n \rightarrow \infty} d\left(x_{n+1}, x_{n}\right)=\lim _{n \rightarrow \infty} d\left(y_{n+1}, y_{n}\right)=0 .
$$

Now, we have to prove that $\left\{x_{n}\right\}$ and $\left\{y_{n}\right\}$ are Cauchy sequences. Suppose that at least one of $\left\{x_{n}\right\}$ and $\left\{y_{n}\right\}$ is not a Cauchy sequence. By Lemma 1.1, there exists an $\epsilon>0$ and two subsequences $\left\{x_{2 k(j)+1}\right\},\left\{x_{2 l(j)+1}\right\}$ of $\left\{x_{n}\right\} ;\left\{y_{2 k(j)+1}\right\},\left\{y_{2 l(j)+1}\right\}$ of $\left\{y_{n}\right\}$ with $j \leq k(j) \leq l(j)$ for all $j \in \mathbb{N}$ such that

$$
\alpha_{j}=d\left(x_{2 k(j)+1}, x_{2 l(j)+1}\right)+d\left(y_{2 k(j)+1}, y_{2 l(j)+1}\right) \geq \epsilon .
$$

We may also assume that

$$
d\left(x_{2 k(j)+1}, x_{2 l(j)}\right)+d\left(y_{2 k(j)+1}, y_{2 l(j)}\right)<\epsilon,
$$

by choosing $l(j)$ to be the smallest number exceeding $k(j)$ for which above holds. From the definition of the function $\psi$, we have

$$
\psi\left(\alpha_{j}\right)=\psi\left(d\left(x_{2 k(j)+1}, x_{2 l(j)+1}\right)+d\left(y_{2 k(j)+1}, y_{2 l(j)+1}\right)\right) \geq \psi(\epsilon)
$$

and

$$
\psi\left(d\left(x_{2 k(j)+1}, x_{2 l(j)}\right)+d\left(y_{2 k(j)+1}, y_{2 l(j)}\right)\right)<\psi(\epsilon) .
$$


From (2.12) and (2.13), and triangle inequality, we obtain

$$
\begin{aligned}
\psi(\epsilon) \leq \psi\left(\alpha_{j}\right)= & \psi\left(d\left(x_{2 k(j)+1}, x_{2 l(j)+1}\right)+d\left(y_{2 k(j)+1}, y_{2 l(j)+1}\right)\right) \\
\leq & \psi\left(d\left(x_{2 k(j)+1}, x_{2 l(j)}\right)+d\left(x_{2 l(j)}, x_{2 l(j)+1}\right)\right. \\
& \left.+d\left(y_{2 k(j)+1}, y_{2 l(j)}\right)+d\left(y_{2 l(j)}, y_{2 l(j)+1}\right)\right) \\
< & \psi(\epsilon)+\psi\left(d\left(x_{2 l(j)}, x_{2 l(j)+1}\right)+d\left(y_{2 l(j)}, y_{2 l(j)+1}\right)\right) .
\end{aligned}
$$

Letting $j \rightarrow \infty$ in the above inequality and using (2.9), (2.12), we get

$$
\psi\left(\alpha_{j}\right)=\psi\left(d\left(x_{2 k(j)+1}, x_{2 l(j)+1}\right)+d\left(y_{2 k(j)+1}, y_{2 l(j)+1}\right)\right) \rightarrow \psi(\epsilon)
$$

Again,

$$
\begin{aligned}
(2.15) \psi\left(\alpha_{j}\right)= & \psi\left(d\left(x_{2 k(j)+1}, x_{2 l(j)+1}\right)+d\left(y_{2 k(j)+1}, y_{2 l(j)+1}\right)\right) \\
\leq & \psi\left(d\left(x_{2 k(j)+1}, x_{2 l(j)+2}\right)+d\left(x_{2 l(j)+2}, x_{2 l(j)+1}\right)\right. \\
& \left.+d\left(y_{2 k(j)+1}, y_{2 l(j)+2}\right)+d\left(y_{2 l(j)+2}, y_{2 l(j)+1}\right)\right) \\
= & \gamma_{2 l(j)+1}+d\left(x_{2 k(j)+1}, x_{2 l(j)+2}\right)+d\left(y_{2 k(j)+1}, y_{2 l(j)+2}\right) \\
\leq & \psi\left(\gamma_{2 l(j)+1}\right)+\psi\left(d\left(x_{2 k(j)+1}, x_{2 k(j)+2}\right)+d\left(x_{2 k(j)+2}, x_{2 l(j)+2}\right)\right. \\
& \left.+d\left(y_{2 k(j)+1}, y_{2 k(j)+2}\right)+d\left(y_{2 k(j)+2}, y_{2 l(j)+2}\right)\right) \\
= & \psi\left(\gamma_{2 l(j)+1}\right)+\psi\left(\gamma_{2 k(j)+1}\right)+\psi\left(d\left(x_{2 l(j)+2}, x_{2 k(j)+2}\right)\right. \\
& \left.+d\left(y_{2 l(j)+2}, y_{2 k(j)+2}\right)\right) .
\end{aligned}
$$

Using (2.1) and (2.15), the above inequality becomes 


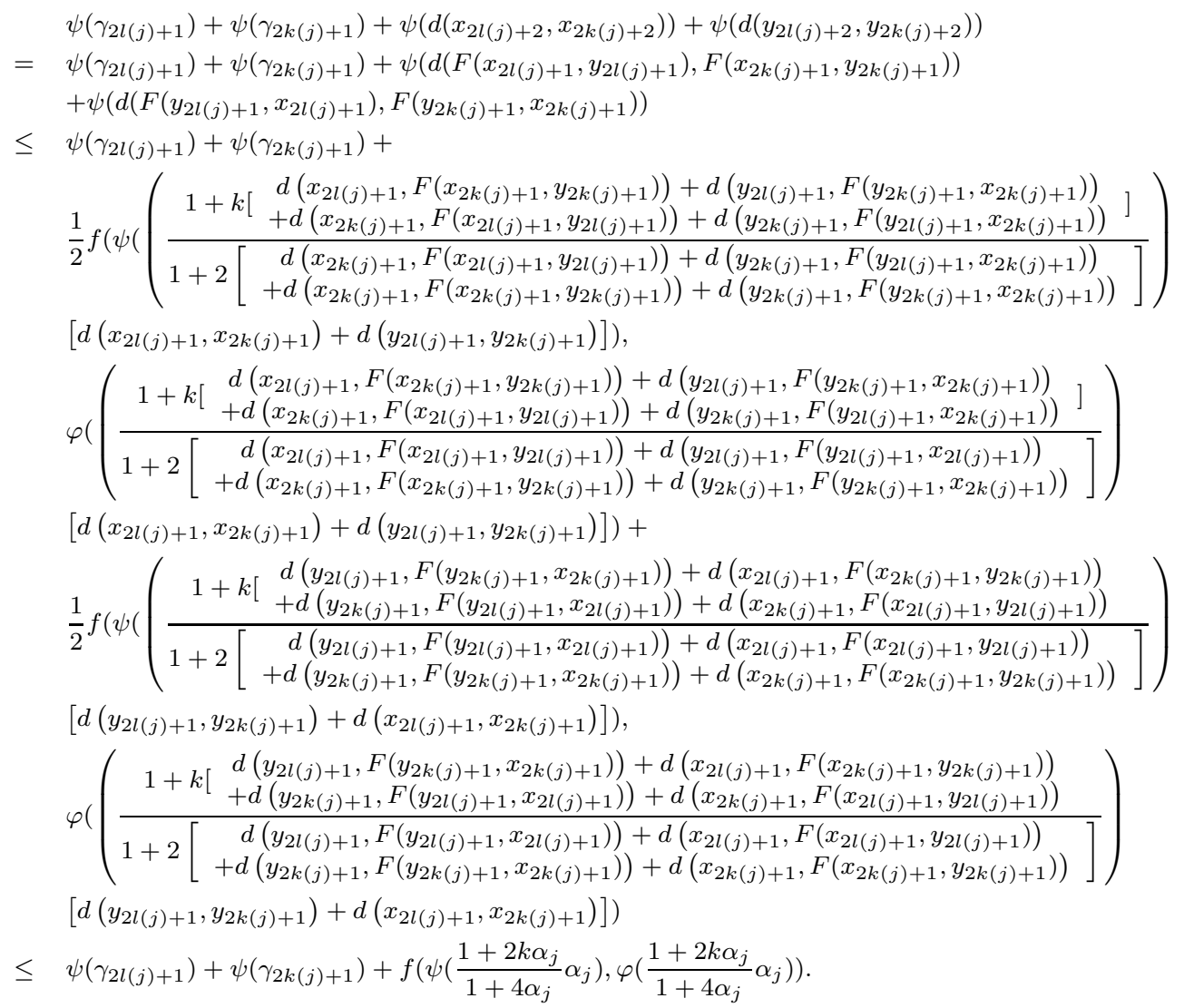

It follows that

$$
\psi\left(\alpha_{j}\right)-\psi\left(\gamma_{2 l(j)+1}\right)-\psi\left(\gamma_{2 k(j)+1}\right) \leq f\left(\psi\left(\frac{\alpha_{j}}{2}\right), \varphi\left(\frac{\alpha_{j}}{2}\right)\right) .
$$

If we take the limit as $j \rightarrow \infty$, we obtain

$$
\psi(\epsilon) \leq f\left(\psi\left(\frac{1+2 k \epsilon}{1+4 \epsilon} \epsilon\right), \phi\left(\frac{1+2 k \epsilon}{1+4 \epsilon} \epsilon\right)\right) \leq \psi\left(\frac{1+2 k \epsilon}{1+4 \epsilon} \epsilon\right) \leq \psi(\epsilon)
$$

So, $\psi\left(\frac{1+2 k \epsilon}{1+4 \epsilon} \epsilon\right)=0$, or $\phi\left(\frac{1+2 k \epsilon}{1+4 \epsilon} \epsilon\right)=0$.Therefore, $\epsilon=0$, giving thereby $\alpha_{j} \rightarrow 0$, which is a contradiction. Thus $\left\{x_{n}\right\}$ and $\left\{y_{n}\right\}$ are indeed Cauchy sequences in the complete metric space $X$. So there exist $x, y \in X$ such that

$$
\lim _{n \rightarrow \infty} x_{n}=x \quad \text { and } \quad \lim _{n \rightarrow \infty} y_{n}=y .
$$

Taking limit both sides in (2.2) and using continuity of $F$, we get

$$
x=\lim _{n \rightarrow \infty} x_{n}=\lim _{n \rightarrow \infty} F\left(x_{n-1}, y_{n-1}\right)=F\left(\lim _{n \rightarrow \infty}\left(x_{n-1}, y_{n-1}\right)\right)=F(x, y)
$$


and

$$
y=\lim _{n \rightarrow \infty} y_{n}=\lim _{n \rightarrow \infty} F\left(y_{n-1}, x_{n-1}\right)=F\left(\lim _{n \rightarrow \infty}\left(y_{n-1}, x_{n-1}\right)\right)=F(y, x) .
$$

Therefore,

$$
x=F(x, y) \text { and } y=F(y, x) .
$$

So $(x, y)$ is a coupled fixed point of $F$.

By choosing $f(s, t)=m s, 0<m<1$ in Theorem 2.1, we obtain the following corollary.

Corollary 2.1. Let $(X, \leq)$ be a partially ordered set and suppose there is a metric $d$ on $X$ such that $(X, d)$ is a complete metric space. Let $F: X \times X \rightarrow X$ be a continuous mapping having the mixed monotone property on $X$. Assume that $F$ satisfies the following condition:

$$
\psi(d(F(x, y), F(u, v))) \leq \frac{m}{2} \psi(\delta(x, y, u, v)[d(x, u)+d(y, v)])
$$

where $\psi \in \Psi, m \in(0,1)$ and a constant $k \in[1,2]$ with

$$
\delta(x, y, u, v)=\frac{1+k[d(x, F(u, v))+d(y, F(v, u))+d(u, F(x, y))+d(v, F(y, x))]}{1+2[d(x, F(x, y))+d(y, F(y, x))+d(u, F(u, v))+d(v, F(v, u))]}
$$

for all $x, y, u, v \in X$ with $x \geq u$ and $y \leq v$. If there exist $x_{0}, y_{0} \in X$ such that

$$
x_{0} \leq F\left(x_{0}, y_{0}\right) \text { and } y_{0} \geq F\left(y_{0}, x_{0}\right) \text {, }
$$

then, $F$ has at least a coupled fixed point $(x, y) \in X \times X$ such that

$$
x=F(x, y) \text { and } y=F(y, x) \text {. }
$$

By putting $\psi(t)=t \quad$ in Corollary 2.1, we obtain the following corollary.

Corollary 2.2. Let $(X, \leq)$ be a partially ordered set and suppose there is a metric $d$ on $X$ such that $(X, d)$ is a complete metric space. Let $F: X \times X \rightarrow X$ be a continuous mapping having the mixed monotone property on $X$. Assume that $F$ satisfies the following condition:

$$
d(F(x, y), F(u, v)) \leq \frac{m}{2} \delta(x, y, u, v)[d(x, u)+d(y, v)]
$$

where $m \in(0,1)$ and a constant $k \in[1,2]$ with

$$
\delta(x, y, u, v)=\frac{1+k[d(x, F(u, v))+d(y, F(v, u))+d(u, F(x, y))+d(v, F(y, x))]}{1+2[d(x, F(x, y))+d(y, F(y, x))+d(u, F(u, v))+d(v, F(v, u))]}
$$

for all $x, y, u, v \in X$ with $x \geq u$ and $y \leq v$.

If there exist $x_{0}, y_{0} \in X$ such that

$$
x_{0} \leq F\left(x_{0}, y_{0}\right) \text { and } y_{0} \geq F\left(y_{0}, x_{0}\right),
$$

then, $F$ has at least a coupled fixed point $(x, y) \in X \times X$ such that

$$
x=F(x, y) \text { and } y=F(y, x) .
$$


Corollary 2.3. Let $(X, \leq)$ be a partially ordered set and suppose there is a metric $d$ on $X$ such that $(X, d)$ is a complete metric space. Let $F: X \times X \rightarrow X$ be a continuous mapping having the mixed monotone property on $X$. Assume that $F$ satisfies the following condition:

$$
d(F(x, y), F(u, v)) \leq \frac{m}{2} \delta(x, y, u, v)[d(x, u)+d(y, v)],
$$

where $m \in(0,1)$ and a constant $k \in[1,2]$ with

$$
\delta(x, y, u, v)=\frac{1+k[d(x, F(u, v))+d(y, F(v, u))+d(u, F(x, y))+d(v, F(y, x))]}{1+2[d(x, F(x, y))+d(y, F(y, x))+d(u, F(u, v))+d(v, F(v, u))]}
$$

for all $x, y, u, v \in X$ with $x \geq u$ and $y \leq v$.

If there exist $x_{0}, y_{0} \in X$ such that

$$
x_{0} \leq F\left(x_{0}, y_{0}\right) \text { and } y_{0} \geq F\left(y_{0}, x_{0}\right),
$$

then, $F$ has at least a coupled fixed point $(x, y) \in X \times X$ such that

$$
x=F(x, y) \text { and } y=F(y, x) .
$$

Theorem 2.2. Let $(X, \leq)$ be a partially ordered set. Suppose there is a metric $d$ on $X$ such that $(X, d)$ is a complete metric space. Let $F: X \times X \rightarrow X$ be a continuous mapping having the mixed monotone property on $X$. Assume that $F$ satisfies the following condition:

$$
\begin{aligned}
d(F(x, y), F(u, v)) \leq & \frac{1}{2} f((\delta(x, y, u, v)[d(x, u)+d(y, v)]) \\
& , \varphi(\delta(x, y, u, v)[d(x, u)+d(y, v)]))
\end{aligned}
$$

where $\varphi \in \Phi_{u}, f \in C$, such that $(I, \varphi, f)$ is monotone and a constant $k \in[1,2]$ with

$$
\delta(x, y, u, v)=\frac{1+k\left[\begin{array}{c}
d(x, F(u, v))+d(y, F(v, u)) \\
+d(u, F(x, y))+d(v, F(y, x))
\end{array}\right]}{1+2\left[\begin{array}{c}
d(x, F(x, y))+d(y, F(y, x)) \\
+d(u, F(u, v))+d(v, F(v, u))
\end{array}\right]}
$$

for all $x, y, u, v \in X$ with $x \geq u$ and $y \leq v$.

If there exist $x_{0}, y_{0} \in X$ such that

$$
x_{0} \leq F\left(x_{0}, y_{0}\right) \text { and } y_{0} \geq F\left(y_{0}, x_{0}\right),
$$

then

a) $F$ has at least a coupled fixed point $(x, y) \in X \times X$ such that

$$
x=F(x, y) \text { and } y=F(y, x) .
$$

b) if $(x, y),(u, v)$ are two distinct coupled fixed points of $F$, then $d(x, u)+$ $d(y, v) \geq \frac{1}{4}$. 
Proof. a) Taking $\psi=I$ in the proof of the first section of Theorem 2.1, then the proof is complete.

b) If there exist two distinct coupled fixed points $(x, y),(u, v)$ of $F$, then

$$
\begin{aligned}
d(x, u)+d(y, v)= & d(F(x, y), F(u, v))+d(F(y, x), F(v, u)) \\
\leq & \frac{1}{2} f((\delta(x, y, u, v)[d(x, u)+d(y, v)]), \varphi(\delta(x, y, u, v) \\
& {[d(x, u)+d(y, v)])) } \\
& +\frac{1}{2} f((\delta(y, x, v, u)[d(y, v)+d(x, u)]), \varphi((\delta(y, x, v, u) \\
& {[d(y, v)+d(x, u)])) } \\
\leq & {[d(x, F(u, v))+d(y, F(v, u))+d(u, F(x, y))+d(v, F(y, x))] } \\
& {[d(x, u)+d(y, v)]+} \\
& {[d(y, F(v, u))+d(x, F(u, v))+d(v, F(y, x))+d(u, F(x, y))] } \\
& {[d(y, v)+d(x, u)] } \\
= & {[d(x, u)+d(y, v)][4 d(x, u)+4 d(y, v)] } \\
= & 4[d(x, u)+d(y, v)]^{2} .
\end{aligned}
$$

Therefore, we obtain that $d(x, u)+d(y, v) \geq \frac{1}{4}$. $(2.1)$.

Now, we will give the following example for the type of mappings which satisfies

Example 2.1. Let $X=\{0,2\}$ and $x \leq y \Leftrightarrow x, y \in\{0,2\}$ and $x \leq y$ where " $\leq$ " be usual ordering then $(X, \leq)$ be a partially ordered set. Define $d: X \times X \rightarrow[0, \infty)$ as follows:

$$
\begin{aligned}
& d(0,2)=4, d(0,0)=d(2,2)=0 \\
& d(a, b)=d(b, a), \forall a, b \in X .
\end{aligned}
$$

Obviously, $(X, d)$ is a complete metric space. Consider now the mapping $F: X \times X \rightarrow X$ given as

$$
F(x, y)=\left\{\begin{array}{l}
0, x=0 \\
2, x=2
\end{array} .\right.
$$

Then $F$ is continuous and has the mixed monotone property. It is obvious that $(0,0),(2,0)$, $(0,2)$ and $(2,2)$ are the coupled fixed points of $F$. In order to show that the contractive condition (2.1) is fulfilled with $\psi(t)=\sqrt[2]{t}, \varphi(t)=\frac{1}{2}$ and $f(s, t)=\frac{s}{1+t}$. Now for $x=2$, $y=0, u=0, v=0$ or $x=2, y=0, u=2, v=2$, we have the following possibilities for values of $(x, y)$ and $(u, v)$ such that $x \geq u$ and $y \leq v$.

Case 1: If we take $(x, y)=(u, v)=r$ where $r=(0,0)$ or $(2,2)$ or $(2,0)$ or $(0,2)$, then $d(F(x, y), F(u, v))=0$. Thus, the inequality (2.1) holds.

Case 2: If we take $(x, y)=(0,0)$ and $(u, v)=(0,2)$, then $d(F(x, y), F(u, v))=0$. Thus, the inequality (2.1) holds.

Case 3: If we take $(x, y)=(2,2)$ and $(u, v)=(0,2)$, then

$$
d(F(x, y), F(u, v))=d(F(2,2), F(0,2))=d(2,0)=4,
$$


and

$$
\begin{aligned}
\delta(x, y, u, v) & =\frac{1+k\left[\begin{array}{c}
d(x, F(u, v))+d(y, F(v, u)) \\
+d(u, F(x, y))+d(v, F(y, x))
\end{array}\right]}{1+2\left[\begin{array}{c}
d(x, F(x, y))+d(y, F(y, x)) \\
+d(u, F(u, v))+d(v, F(v, u))
\end{array}\right]} \\
& =\frac{1+k[d(2,0)+d(2,2)+d(0,2)+d(2,2)]}{1+2[d(2,2)+d(2,2)+d(0,0)+d(2,2)]} \\
& =1+8 k .
\end{aligned}
$$

Therefore, the inequality (2.1) holds.

Case 4: If we take $(x, y)=(2,0)$ and $(u, v)=(0,0)$, then

$$
d(F(x, y), F(u, v))=d(F(2,0), F(0,0))=d(2,0)=4,
$$

and

$$
\begin{aligned}
\delta(x, y, u, v) & =\frac{1+k\left[\begin{array}{c}
d(x, F(u, v))+d(y, F(v, u)) \\
+d(u, F(x, y))+d(v, F(y, x))
\end{array}\right]}{1+2\left[\begin{array}{c}
d(x, F(x, y))+d(y, F(y, x)) \\
+d(u, F(u, v))+d(v, F(v, u))
\end{array}\right]} \\
& =\frac{1+k[d(2,0)+d(0,0)+d(0,2)+d(0,0)]}{1+2[d(2,2)+d(0,0)+d(0,0)+d(0,0)]} \\
& =1+8 k .
\end{aligned}
$$

Or, taking $(x, y)=(2,0),(u, v)=(0,2)$, we have

$$
d(F(x, y), F(u, v))=d(F(2,0), F(0,2))=d(2,0)=4,
$$

and

$$
\begin{aligned}
\delta(x, y, u, v) & =\frac{1+k\left[\begin{array}{c}
d(x, F(u, v))+d(y, F(v, u)) \\
+d(u, F(x, y))+d(v, F(y, x))
\end{array}\right]}{1+2\left[\begin{array}{c}
d(x, F(x, y))+d(y, F(y, x)) \\
+d(u, F(u, v))+d(v, F(v, u))
\end{array}\right]} \\
& =\frac{1+k[d(2,0)+d(0,2)+d(0,2)+d(2,0)]}{1+2[d(2,2)+d(0,0)+d(0,0)+d(2,2)]} \\
& =1+16 k .
\end{aligned}
$$

Therefore, the inequality (2.1) holds.

Case 5: If we take $(x, y)=(1,0)$ and $(u, v)=(2,2)$, then

$$
d(F(x, y), F(u, v))=d(F(2,0), F(2,2))=d(2,2)=0,
$$

that is, the inequality (2.1) holds.

Thus all the conditions of Theorem 2.1 are satisfied. And $F$ has four distinct coupled fixed points $(0,0),(2,0),(0,2)$ and $(2,2)$ in $X \times X$. 


\section{R E F E R E N C E S}

1. Agarwal, R.P., El-Gebeily, MA, O 'Regan, D., Generalized contractions in partially ordered metric spaces, Appl. Anal. 87(2008),1-8.

2. Ansari, A. H., Note on $\varphi-\psi$-contractive type mappings and related fixed point, The 2nd Regional Conference on Mathematics and Applications, PNU, September 2014, pages $377-380$

3. Babu G.V.R. and Sailaja, P.D. , A Fixed Point Theorem of Generalized Weakly Contractive Maps in Orbitally Complete Metric Spaces, Thai Journal of Mathematics, 9(1)(2011), 1-10.

4. Berinde, V., Generalized coupled fixed point theorems for mixed monotone mappings in partially ordered metric spaces, Nonlinear Analysis,74 (2011),7347-7355.

5. Bhaskar. T.G. and Lakshmikantham, V., Fixed point theorems in partially ordered metric spaces and applications, Nonlinear Anal.,65(2006),1379-1393 .

6. M. S. Khan, M. Swaleh, and S. Sessa, Fixed point theorems by altering distances between the points, Bull. Austral.Math.Soc., 30(1)(1984),1-9.

7. Nieto, J.J. and Lopez, R.R., Contractive mapping theorems in partially ordered sets and applications to ordinary differential equations, Order,22 (2005), 223-239.

8. Nieto, J.J. and Lopez, R.R., Existence and uniqueness of fixed point in partially ordered sets and applications to ordinary deferential equations, Acta Math. Sinica Engl. Ser. 23(12)(2007),2205-2212.

9. Ran, ACM, and Reurings, MCB, A fixed point theorem in partially ordered sets and some applications to matrix equations, Proc. Amer. Math. Soc. 132(2004), 1435-1443.

10. O'Regan, D. and Petrusal, A., Fixed point theorems for generalized contractions in ordered metric spaces, J.Math. Anal. Appl., 341(2008), 241-1252.

11. Yildirim, I., A new type of coupled fixed point theorem in partially ordered complete metric space, Journal of Mathematical Analysis, 7(3)(2016),58-65.

Arslan Hojat Ansari

Department of Mathematics, Karaj Branch, Islamic Azad University, Karaj, Iran analsisamirmath2@gmail.com

Murat Ozdemir

Faculty of Science, Department of Mathematics, Ataturk University

P.O. Box 25240, Erzurum, Turkey

mozdemir@atauni.edu.tr

Mohammad S. Khan

Department of Mathematics \& Statistics, Sultan Qaboos University

P. O. Box 36, Al-Khod 123, Muscat, Sultanate of Oman

mohammad@squ. edu.om 
Isa Yildirim

Faculty of Science, Department of Mathematics, Ataturk University P.O. Box 25240, Erzurum, Turkey

isayildirim@atauni.edu.tr 